\title{
Unplanned School Closures in the United States: Evaluation of Economic and Social Costs and Consequences for Students' Families
}

\author{
Yenlik Zheteyeva*, Margaret Coleman, JianRong Shi, Hongjiang Gao, Amra Uzicanin and \\ Jeanette J. Rainey
}

DGMQ, CDC, Atlanta, GA, USA

\section{Introduction}

The Centers for Disease Control and Prevention (CDC) recommends implementing early targeted school closures as one of the front-line interventions to slow progression of a severe influenza pandemic before appropriate vaccine becomes available. However, prolonged school closures may impose unintended economic and social costs and consequences to students' families. These costs and consequences have not been carefully evaluated. To better understand this unintended impact, we conducted five investigations of unplanned school closures lasting $\geq 4$ school days implemented for various reasons from August 2012 through May 2013. Each closure was investigated separately as a public health evaluation. School closures implemented for reasons other than pandemic influenza may serve as a proxy to pandemic-related closures. Our findings can inform updates to CDC's pandemic preparedness guidance.

\section{Methods}

We investigated unplanned school closures in an urban-rural school district (20 schools, 14,368 students, closed to prepare for anticipated hurricane) in a coastal region of Mississippi, a rural school district (3 schools, 524 students, closed for high influenza-related absenteeism) in Kentucky, an urban elementary school in Maine (427 students, closed due to electric fire), a school district (3 schools, 204 students, closed due to high influenza-related absenteeism) in a suburban community in Colorado, and a rural school district (4 schools, 1,534 students, closed to prepare for flooding) in Illinois, with overall enrollment of 17,057 students. We distributed paper questionnaires about the unplanned school closure to all parents and guardians via students at each affected school. We requested completion of only one questionnaire per household. We evaluated perceptions of difficulty arising from the school closure, including difficulty finding alternative childcare options, additional expenses incurred during the school closure, and difficulties related to lost access to subsidized school lunches.

\section{Results}

Questionnaires were completed by 2,700 households (accounting for 4,935 students), with household response rate ranging from 25$38 \%$ in different locations. Median duration of school closures was 5 work days (range: $4-7$ days). The majority $(82.5 \%)$ of responding households were those affected by the Mississippi unplanned school closure. The median proportion of households reporting that school closure caused them difficulty was $25 \%$, ranging from $18 \%$ in the Illinois school district to $98 \%$ in the elementary school in Maine. The most commonly reported difficulty (reported as one of the top two reasons from at least three sites) included uncertainty about the duration of the school closure and difficulty with arranging childcare. The median proportion of households reporting difficulty providing food to families due to lost access to free or reduced price school lunch program during the school closure was quite low, $8 \%$ (ranging from $3 \%$ in Colorado to $18 \%$ in Mississippi). Approximately $25 \%$ of all respondents reported incurring additional expenses during the closures, with a median daily amount of $\$ 33$ (range: $\$ 14.3-\$ 53.7$ ). The most frequently reported childcare provider was a non-working adult household member in all survey communities except Maine, where a non-household adult was reported as the most frequent provider. Households where all adults work outside of the home were significantly more likely to report difficulty arranging childcare ( $p$ $<0.001$ in Kentucky).

\section{Conclusions}

One of the primary challenges for families during unplanned school closures is making emergency childcare arrangements. While the most frequently childcare is provided by a non-working household member, this option is not available for families where all adults are employed full-time. Although disruption of subsidized school lunch program did not appear as a difficulty for the majority of eligible families during these school closures, more research might be needed on closures that last longer than 7 days.

\section{Keywords}

School closures; Pandemic influenza; Economic and social impact

\section{*Yenlik Zheteyeva}

E-mail: igg0@cdc.gov 